\title{
Leg Stiffness and Expertise in Men Jumping
}

\author{
GUILLAUME LAFFAYE ${ }^{1}$, BENOÎT G. BARDY ${ }^{1,2}$, and ALAIN DUREY ${ }^{3}$ \\ ${ }^{1}$ Center for Research in Sport Sciences, University Paris XI, Paris, FRANCE; ${ }^{2}$ Institut Universitaire de France, Paris, \\ FRANCE; and ${ }^{3}$ Laboratory of Aerodynamics and Biomechanics, University of the Mediterranean, Marseille, FRANCE
}

\begin{abstract}
LAFFAYE, G., B. G. BARDY, and A. DUREY. Leg Stiffness and Expertise in Men Jumping. Med. Sci. Sports Exerc., Vol. 37, No. 4, pp. 536-543, 2005. Purpose: The aim of the present study is to investigate: a) the leg spring behavior in the one-leg vertical jump, b) the contribution of impulse parameters to this behavior, and c) the effect of jumping expertise on leg stiffness. Methods: Four categories of experts (handball, basketball, volleyball players, and Fosbury athletes), as well as novice subjects performed a run-and-jump test to touch a ball with the head. Five experimental conditions were tested from 55 to $95 \%$ of the maximum jump height. Kinematic and kinetic data were collected using six cameras and a force plate. Results: The mechanical behavior of the musculoskeleton component of the human body can be modeled as a simple mass-spring system, from which leg stiffness values can be extracted to better understand energy transfer during running or jumping. The results indicate that leg stiffness (mean value of 11.5 $\mathrm{kN} \cdot \mathrm{m}^{-1}$ ) decreased with jumping height. Leg shortening at takeoff also increased with jumping height, whereas contact time decreased $(-18 \%)$. No difference was found between experts and novices for leg stiffness. However, a principal components analysis (PCA) indicated the contribution of two main factors to the performance. The first factor emerged out of vertical force, stiffness, and duration of impulse. The second factor included leg shortening and jumping height. Conclusion: Differences between experts and novices were observed in terms of the contribution of leg stiffness to jump height, and more importantly, clear differences existed between experts in jumping parameters. The analysis performed on the sport categories indeed revealed different jumping profiles, characterized by specific, sport-related impulse parameters. Key Words: SPRING-MASS MODEL, HOPPING, NOVICES, EXPERTS
\end{abstract}

$\mathrm{D}$ uring running or bouncing, bipedal animals use musculoskeletal springs to alternately store and restitute elastic energy (5). In the run-and-jump transition, muscles, tendons, and ligaments collectively behave like a linear spring, store kinetic energy during the run-up, and restitute it during the jump in the form of potential gravitational energy. Simple mass-spring systems consisting of a single linear spring representing the leg, attached to a point summarizing body mass, have been shown to capture efficiently the mechanics of running or jumping $(4,10,18-20)$.

The stiffness of the spring (i.e., leg stiffness or $\mathrm{k}_{\mathrm{leg}}$ ) can be defined in different ways and at different levels of analysis. First, $\mathrm{k}_{\mathrm{leg}}$ can be understood as an analytic mechanical concept, and calculated by the sum of all joint stiffnesses. This method allows the assessment of the internal behavior of the joints (12). Second, $\mathrm{k}_{\mathrm{leg}}$ can be approached as a general mechanical concept, and computed by the ratio of the maximum vertical ground reaction force on leg shortening $(15,19)$. Another recent method to estimate $\mathrm{k}_{\text {leg }}$ intro-

\footnotetext{
Address for correspondence: Guillaume Laffaye, Center for Research in Sport Sciences, University Paris XI, Bât 335, 91405 Orsay Cedex, France; E-mail: cglaffaye@aol.com.

Submitted for publication June 2004.

Accepted for publication December 2004.
}

0195-9131/05/3704-0536

MEDICINE \& SCIENCE IN SPORTS \& EXERCISE E

Copyright (C) 2005 by the American College of Sports Medicine

DOI: 10.1249/01.MSS.0000158991.17211.13 duces wobbling masses (20), allowing the prediction of a more realistic passive peak, due to the soft tissues of the leg.

Up until now, two main jumping behaviors have been investigated for accessing $\mathrm{k}_{\mathrm{leg}}$ : hopping in place at a given frequency, and the drop jump. During hopping, $\mathrm{k}_{\mathrm{leg}}$ increases with hopping frequency and/or hopping height $(9,14)$, and is modulated to accommodate the changes due to the compliance of the support surface (11). Here $\mathrm{k}_{\text {leg }}$ primarily depends on ankle stiffness (12), and changes as a function of gender (15). During the drop jump, $\mathrm{k}_{\text {leg }}$ appears to be sensitive to jumping instructions, and is scaled to contact time (3).

The dynamics of $k_{\text {leg }}$ in one-leg vertical jumps (such as in high jumping) is still unknown. Only one study has investigated the contribution of $\mathrm{k}_{\mathrm{leg}}$ to the performance in (horizontal) long jumping (20). The complementary findingsthat a minimum value of stiffness is required for an optimal performance $\left(\mathrm{k}_{\operatorname{leg}}=16.2 \mathrm{kN} \cdot \mathrm{m}^{-1}\right)$, and that for any given stiffness there is an optimum angle of attack-suggest that $\mathrm{k}_{\text {leg }}$ is a decisive, although not unique, variable contributing to jumping performance. However, vertical and horizontal jumps differ in many mechanical ways (2), and the contribution of $k_{\text {leg }}$ to performance in high jumping may differ. The goal of the present study is to investigate: a) the leg spring behavior in the one-leg vertical jump, b) the contribution of impulse parameters to this behavior, and c) the effect of jumping expertise on $\mathrm{k}_{\mathrm{leg}}$.

\section{METHODS}

Twenty-three male subjects participated in this study. They were grouped in five different categories: four Fos- 
TABLE 1. Subject characteristics (mean values \pm SD).

\begin{tabular}{|c|c|c|c|c|c|}
\hline $\begin{array}{l}\text { Type of } \\
\text { Expertise }\end{array}$ & $\begin{array}{l}\text { Fosbury } \\
\text { Athletes }\end{array}$ & Novices & Volleyball & Handball & Basketball \\
\hline
\end{tabular}

bury athletes (mean jump performance of $2.08 \pm 0.04 \mathrm{~m}$ ), five volleyball players, four handball players, five basketball players (all playing at the national level), and five novices in jumping sports. The distinction between novices and experts was based on two main factors. Long experience in jumping practice in their respective sport was a first criterion characterizing the expert population. All experts had a minimum of $5 \mathrm{yr}$ of practice in jumping, with a minimum of one training session every week. The second factor was the competitive level of practice. A minimum of $5 \mathrm{yr}$ at the national level (French championship) was required to be tested in this study. All experts in all tested sports (volleyball, Fosbury, basketball, and handball) met this criterion. Novices were subjects with no consistent experience or training in jumping. They had, of course, never participated in any jumping competition.

In accordance with the ethical principles of human research experiments issued by the University of Paris XI, each volunteer signed an informed consent statement after receiving oral and written description of the procedures as well as the risks and benefits of participation of this study. Their main anthropometrical characteristics are summarized in Table 1.

\section{Task and Procedure}

Baseline test. All participants began with a one-leg maximum vertical jump test. In that test, participants were asked to run over $5 \mathrm{~m}$, jump with one foot, and touch a target with the top of the head (Fig. 1). The target was a smooth ball $(25 \mathrm{~cm}$ in diameter) attached to the end of a thread going over a pulley that could be manipulated by the experimenter. The test was repeated until the maximum jumping height was found. The maximum height was assessed with a precision of $\pm 1 \mathrm{~cm}$ by increasing the target of $2 \mathrm{~cm}$ when the lower target was touched with the head, and was used as a reference for designing the five conditions of the experimental session. The test stopped when the subject failed to touch the target.

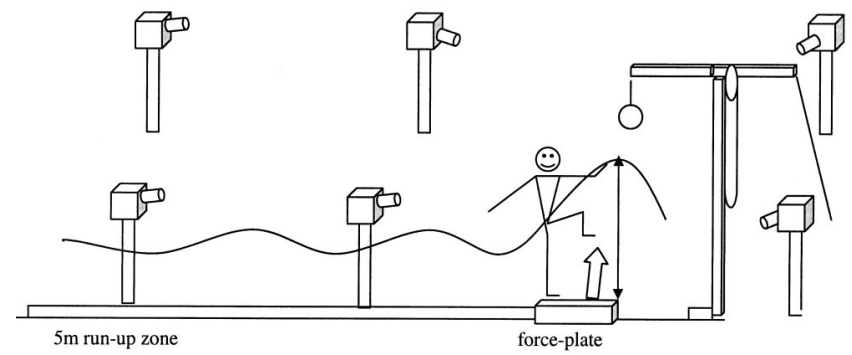

FIGURE 1-Task and apparatus for the baseline and experimental tests.
Experimental test. After a 10-min rest, the experimental test started. Each trial was considered successful when there was a "smooth" contact of the head with the ball. Five experimental conditions were tested, corresponding to 55 , $65,75,85$, and $95 \%$ of the maximum height obtained during the baseline test. There were five trials per condition, presented in a randomized order, and counterbalanced over participants to avoid order effects. Each experimental session lasted about $30 \mathrm{~min}$, including a 1-min rest every five trials. Impulse parameters were recorded with a force plate during the experimental session, as described below.

\section{Data Collection and Analysis}

We collected the kinetics and the kinematics of each experimental trial. For the kinetic data, a $40 \times 40 \mathrm{~cm}$ AMTI OR 6-5 force plate was positioned at the end of the run-up and at the same level. The horizontal distance between the force plate and the vertical plane containing the ball was adjusted for each participant so that the last impulse could be produced in the middle of the force plate in a comfortable position. The force plate sampling frequency was $600 \mathrm{~Hz}$. The vertical ground reaction force was extracted from the impulse signal at takeoff. For the kinematic data, the motion of body segments was recorded in 3D with a 6-MCAMcameras 640-VICON motion analysis system, at a sampling rate of $120 \mathrm{~Hz}$. Thirty-five reflecting markers were positioned on various parts of the body (top of the head $(N=4)$; shoulders $(N=2)$; wrists $(N=2)$; forearms $(N=2)$; elbows $(N=2)$; arms $(N=2)$; sternum, C7, back, and hip $(N=4)$; thighs and knees $(N=2)$; shins $(N=2)$; ankles $(N=2)$; heels $(N=2)$; and toes $(N=2)$. A 3D-32 segment-human body model was reconstructed, and the position and motion of the body center of mass were computed using Chandler et al.'s (6) biomechanical model.

A spring-mass model, which consisted of a mass attached to a single linear massless spring, was used to analyze the mechanics of the jump $(12,19)$. We considered leg stiffness as a general mechanical concept that captures the global behavior of the body during the jump. During the contact phase, leg length was defined as the distance between the body center of mass and the ball of the foot, considered as the rotational center of the system during ground contact. Leg stiffness was defined as the ratio of the maximal ground reaction force $\mathrm{F}_{\max }$ during the active peak to the leg shortening $\Delta r$ (3) at the time of maximum leg shortening (Fig. 2), namely:

$$
\mathrm{k}_{\operatorname{leg}}=\mathrm{Fmax} / \Delta \mathrm{rmax}
$$




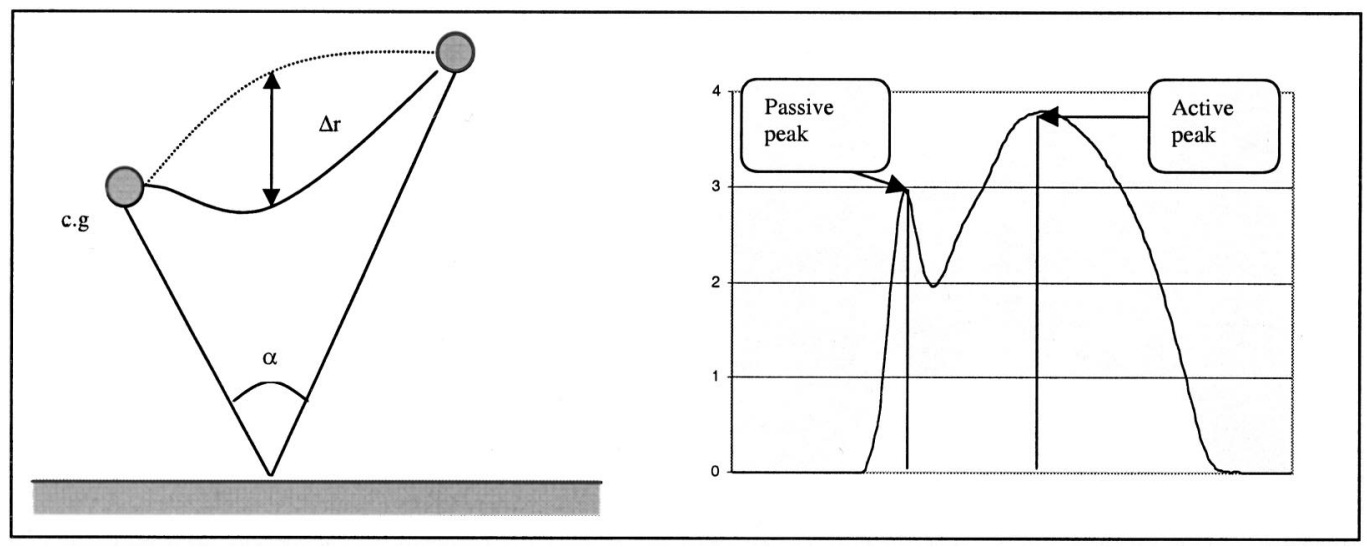

FIGURE 2-Mass-spring model for high jump. $\Delta \mathrm{r}$ refers to the current shortening of the leg, and $\alpha$ to the angle swept by the leg during the ground contact phase. The position of the center of mass (c.g.) is higher at takeoff due to the constraints of the vertical jumping task. The maximum ground reaction force is calculated from the active peak.

\section{Variables and Takeoff Parameters}

For each trial, the following variables were computed:

Constant and absolute errors (CE and $\mathrm{AE}$ ). Because the goal of the task was to achieve a smooth contact between head and target, the difference between the position of the top of the head and the target when both crossed the same frontal plane was computed and used to obtain CE and AE. A positive $\mathrm{CE}$ indicated a contact with the target, and a negative $\mathrm{CE}$ indicated no contact:

$$
C E=\frac{\sum_{i=1}^{N}\left(x_{i}\right)}{N} \quad A E=\frac{\sum_{i=1}^{N}\left|x_{i}\right|}{N}
$$

where $\mathrm{x}$ represents the value of the variable, and $N$ the number of values.

Maximal vertical ground reaction force $\left(F_{\text {max }}\right)$. $F_{\max }$ was obtained from the force plate values by direct reading capturing by the software 3 Dvision. $\mathrm{F}_{\max }$ was calculated at the maximum value of the active peak of the force-time curve. Ground reaction force was normalized to each subject's body weight.

Ground contact time (CT). This was the time of contact with the ground on the last step, that is, between touchdown and takeoff.

Angle swept by the center of mass during the ground contact phase $(\alpha)$, measured from touchdown to takeoff. From geometric considerations, and because the takeoff angle is constant, an equation for calculating $\alpha$ from the time of foot contact with the ground (CT), the forward speed $(\mathrm{u})$, and the initial leg length $\left(\mathrm{L}_{0}\right)$ was obtained (19):

$$
\Delta L=\Delta r+L_{0}(1-\cos \alpha)
$$

\section{RESULTS}

The absolute error for each participant and each condition was less than $10 \%$. We therefore decided to analyze all trials. However, technical problems with the reconstruction process (glitches, phantom markers, etc.) prevented us from reconstructing $8 \%$ of the trials. In total, 470 trials were reconstructed, processed, and analyzed. All results are summarized in Figure 3.

Constant and absolute errors. Mean value of $\mathrm{CE}$ was $-0.42 \mathrm{~cm}$. The mean ratio of $\mathrm{CE}$ was about $1 \%$ of the required height, suggesting that the regulation of the jump was successful. A two-way ANOVA (expertise $\times$ height) with repeated measures on the second factor revealed a significant effect on CE for height $[F(4,64)=17.49, P<$ 0.05 ], with lower heights being slightly overestimated and larger heights slightly underestimated. However, $\mathrm{AE}$ (mean of $3.3 \mathrm{~cm}, \mathrm{SD}=1.2$ ) did not differ between conditions $[F(4,64)=2.44]$, and represented about $4 \%$ of the participants' spring.

Jumping height. The vertical displacement of the center of mass during the aerial phase varied between $76 \mathrm{~cm}$ for the Fosbury athletes $(82 \mathrm{~cm}$ was the highest value) and 58 $\mathrm{cm}$ for the novices $(52 \mathrm{~cm}$ was the lowest value). Overall, the mean displacement was $67 \mathrm{~cm}(\mathrm{SD}=7.5)$. A two-way ANOVA (expertise $\times$ height) revealed a significant main effect for height $[F(4,64)=75.66 ; P<0.01]$ and expertise $[F(4,16)=3.55 ; P<0.05]$. The interaction was not significant $[F(16,64)<1]$. Planned post hoc comparisons revealed a significant difference between the four groups of experts on one hand and the novices on the other hand $[F(1,16)=4.81 ; P<0.05]$.

Maximal ground reaction force. The mean absolute value of $\mathrm{F}_{\max }$ was $2310 \mathrm{~N}(\mathrm{SD}=334 \mathrm{~N})$, and ranged from 1102 to $3044 \mathrm{~N}$. The mean relative value of $\mathrm{F}_{\text {rel }}\left(\mathrm{F}_{\text {rel }}=\right.$ $\mathrm{F}_{\max } / \mathrm{mg}$ ) was $3.21 \times$ body weight $(\mathrm{SD}=0.41)$, and ranged from 2.03 to $3.89 \times$ body weight. Using a normalized value of the force variable $\left(\mathrm{F}_{\mathrm{rel}}=\mathrm{F}_{\max } / \mathrm{mg}\right)$, the relative force was found to be higher for the Fosbury athletes (3.5 times the body weight) and lower for the volleyball players ( 2.8 times the body weight). A two-way ANOVA (expertise $\times$ height) performed on $\mathrm{F}_{\text {rel }}$ showed a significant effect of height $[F(4,64)=11.50]$, indicating larger values of $F_{\text {rel }}$ as height was increased.

Ground contact time (CT). Mean ground contact time was $255 \mathrm{~ms}$ ( $\mathrm{SD}=31 \mathrm{~ms})$, with lowest and highest values 

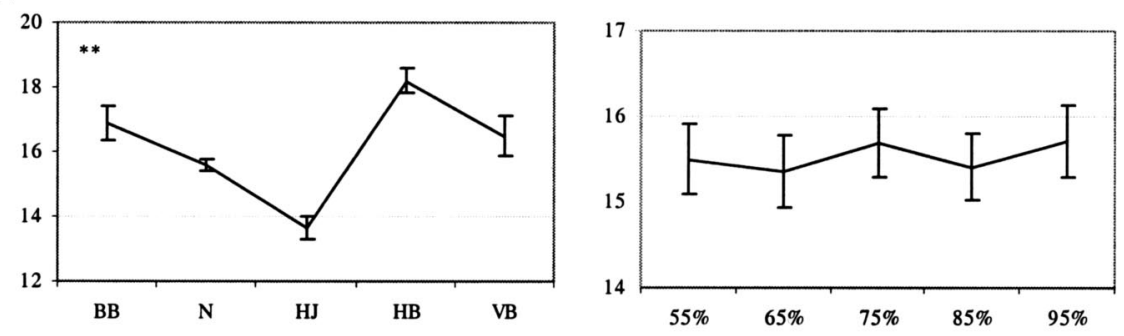

Angle swept by the leg $\left(^{\circ}\right)$
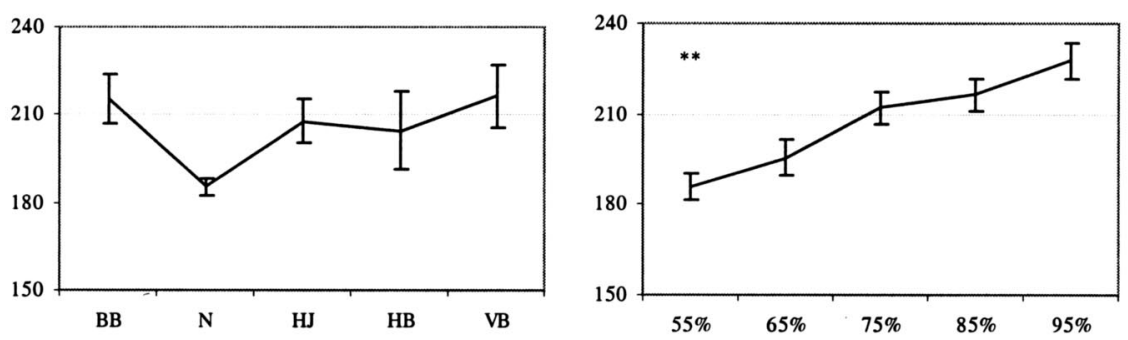

Leg shortening (mm)
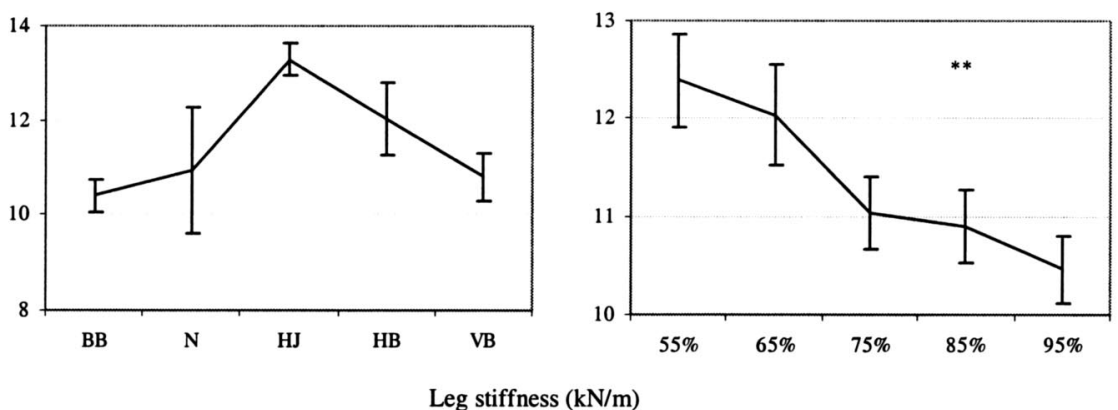

FIGURE 3-Effects of expertise (left) and height (right) on jump parameters and leg stiffness: BB, basketball; N, novices; HJ, Fosbury athletes; HB, handball; VB, volleyball. The percentage represents the five experimental conditions (respectively from 55 to $95 \%$ of the maximum individual height). $* P<0.05 ; * * P<0.01$ (general factor effect).

ranging between 190 and $367 \mathrm{~ms}$. A height $\times$ expertise ANOVA performed on CT indicated no significant effect for expertise $[F(4,16)<1]$. A main effect of height was found $[F(4,64)=6.09, P<0.01]$, with mean values decreasing from 265 to $251 \mathrm{~ms}$ as height was increased from 55 to $95 \%$.

Angle swept by the leg. $\alpha$ had a mean value of $16^{\circ}$ $\left(\mathrm{SD}=2.17^{\circ}\right)$ across heights and types of expertise, with values ranging from 10 to $20^{\circ}$. A height $\times$ expertise ANOVA yielded no significant effect of height $[F(4,64)=$ $1.03, \mathrm{NS}]$, suggesting that $\alpha$ did not contribute in itself in a crucial way to the performance. However, a significant effect was found for expertise $[F(4,16)=7.48, P<0.01]$ that revealed interesting differences between groups. A post hoc Scheffe test indicated a significant difference between the Fosbury athletes and the three other sports categories: basketball, volleyball, and handball $(P<0.05)$. The angle swept by the leg was lower in this study than the value found $(7,8)$ in high jumping $\left(16\right.$ vs $\left.20^{\circ}\right)$, which could be explained by the short run-up of our task. The increase in this angle when the target height increases was observed for the three categories: $+3.7^{\circ}$ for the handball players, $+2.91^{\circ}$ for the Fosbury athletes, and $+1.2^{\circ}$ for the basketball players between block 1 and block 5. Thus, a common motor strategy consisting of increasing the angle swept by the leg appears to exist.

On the other hand, a second profile appears for the novices and the volleyball players, with no change in $\alpha$ between low and high jumps. In Figure 4 we can observe that for the volleyball players this angle does not vary with the blocks, but for the novices this angle varies randomly.

Leg shortening. Following Seyfarth et al. (20), we measured the leg shortening $\Delta \mathrm{r}$ at the maximum compression of the spring (see Fig. 2). $\Delta \mathrm{r}$ had a mean of $20.4 \mathrm{~cm}$ (SD $=3.2 \mathrm{~cm}$ ) across height and expertise, with values ranging between 12.2 and $30 \mathrm{~cm}$. A height $\times$ expertise ANOVA performed on $\Delta \mathrm{r}$ revealed a main effect of height $[F(4,64)=$ $36.86, P<0.01$ ], indicating a $22 \%$ increase in $\Delta \mathrm{r}$ as target height increased from 55 to $95 \%$. The type of expertise did not affect $\Delta \mathrm{r}, F(4,16)=2.09$, and these two effects were independent, as suggested by the height $\times$ expertise interaction that failed to reach significance, $F(16,64)<1$. In 

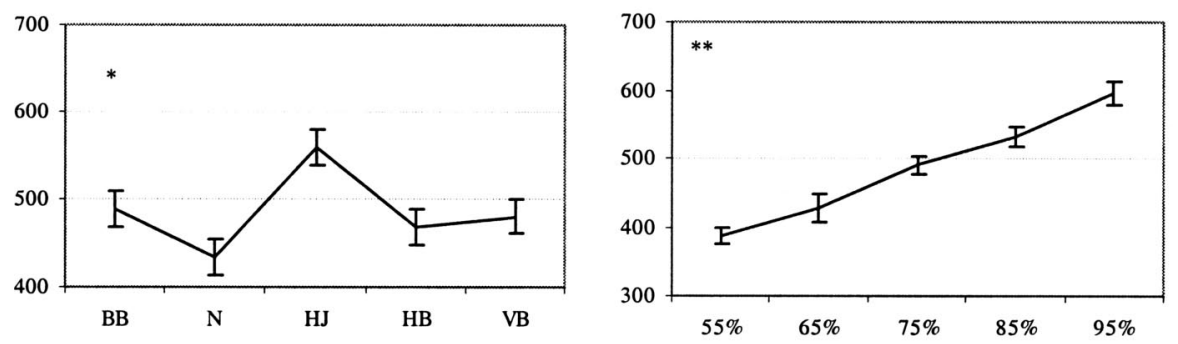

Jump height (mm)
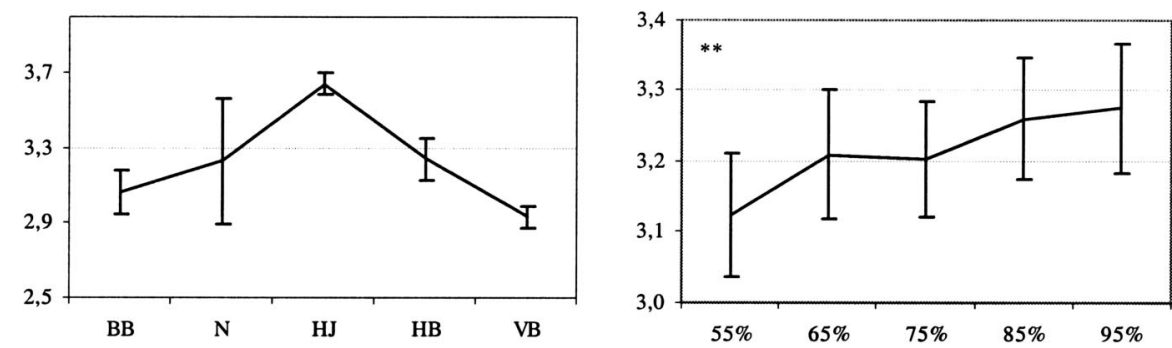

Normalised ground reaction force (F/mg)
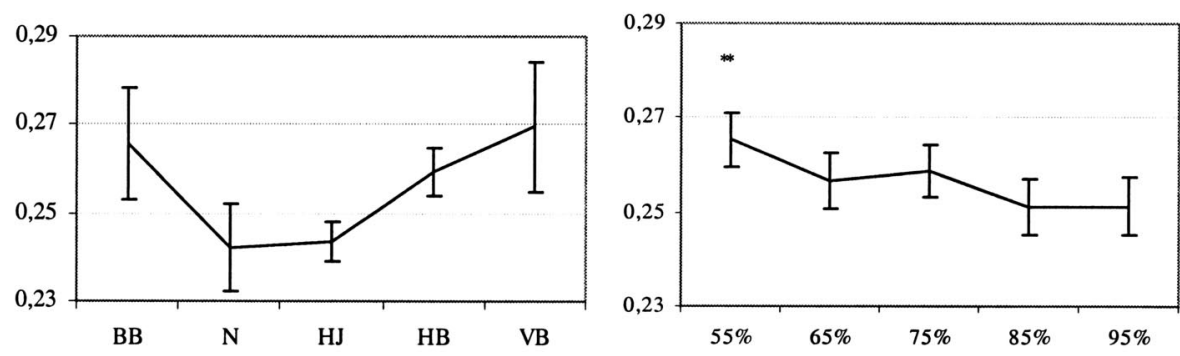

Ground contact time (s)

FIGURE 3-(continued)

sum, increasing the compressing of the spring as target height increased was a natural behavior shared by all experts.

Leg stiffness. The stiffness of the leg $(\mathrm{k})$ is the ratio between $\mathrm{F}_{\max }$ and the maximal $\Delta \mathrm{r}$ (equation 1), and is thus not independent on these two variables. Mean values of $\mathrm{k}_{\mathrm{leg}}$ were $12.3,12,11,10.8$, and $10.4 \mathrm{kN} \cdot \mathrm{m}^{-1}$ in the five experimental conditions. $\mathrm{k}_{\mathrm{leg}}$ differed between height conditions $[F(4,64)=20.01, P<0.01]$, revealing an interesting decrease in leg stiffness as the target height was increased without showing any difference between groups.

Principal components analysis (PCA). The goal of the PCA was to determine whether (and which of) the interdependent variables could be reduced to a smaller number of factors. The PCA was performed only in the $95 \%$ condition. The number of components was determined by the number of eigenvalues higher than 1, using an orthogonal rotation of the variables in the original data set (normalized VARIMAX). Two factors emerged out of the PCA (Table 2), accounting for $76 \%$ of the total variance. The first component, which accounted for $44.54 \%$ of the variance, associated $\mathrm{k}_{\mathrm{leg}}(-0.750), \mathrm{F}_{\text {rel }}(-0.904)$, and CT (0.794). Negative loading of both force and stiffness indicated a relationship between impulse duration and the possibility of increasing leg stiffness using ground reaction force. This negative relation echoes the analysis performed by Arampatzis et al. (3), which showed an increase in leg stiffness due to a decrease in contact time. The second factor, accounting for $31.64 \%$ of the total variance, associated $\Delta \mathrm{r}$ (0.883) and jumping height (0.925). It indicated that compressing the leg's spring was a common strategy adopted by all participants to increase jumping height.

Plotting mean individual scores on the two principal components allowed us to investigate potential differences between experts. In Figure 5, the X-axis corresponds to the first principal component, bringing $\mathrm{CT}, \mathrm{k}_{\mathrm{rel}}$, and $\mathrm{F}_{\mathrm{rel}}$ together. This profile mostly characterized the volleyball jumpers, with high-factor scores ranging from 2 to 3.5. Basketball players were also close to this profile, with lower factor scores ranging from 0 to 1.5. The y-axis in Figure 5 corresponds to the second principal component. High jumpers revealed high-factor scores on this axis, with values 


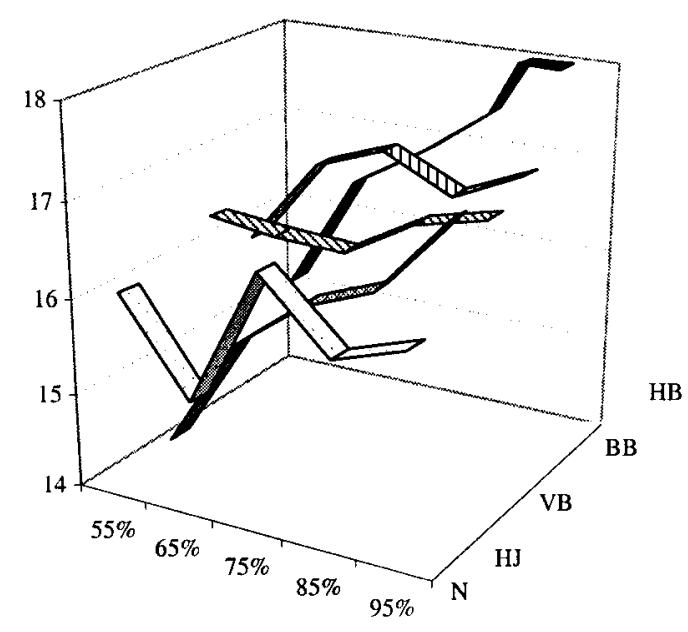

FIGURE 4-Variation of the angle swept by the leg $\left({ }^{\circ}\right)$ with the height conditions and between-sports categories. BB, basketball; N, novices; HJ, Fosbury athletes; HB, handball; VB, volleyball.

ranging from 2.5 to 3.5 , indicating a decisive leg shortening. Novices revealed negative factor scores (from 0 to 3 ), indicating that small jumps were associated with a small $\Delta \mathrm{r}$.

\section{DISCUSSION AND CONCLUSION}

The aim of this study was to assess the contribution for different jumping experts of leg stiffness to jumping height. Overall, the task was performed in a satisfactory way by all categories, without differences in absolute or constant errors $(\mathrm{CE}<1 \%$ and $\mathrm{AE}<4 \%)$. The experts $(69 \mathrm{~cm})$ jumped significantly higher than the novices $(58 \mathrm{~cm})$. Normalized ground reaction forces and leg shortening increased with imposed height, whereas contact time and leg stiffness decreased. The angle swept by the leg did not change with height (about $16^{\circ}$ ), but discriminated experts from novices.

\section{Contribution of leg stiffness to jumping height.} The mean value of $\mathrm{k}_{\text {leg }}$ found in our run-and-jump task (mean of $11.5 \mathrm{kN} \cdot \mathrm{m}^{-1}$ ) did not greatly differ from that found in running $\left(12-15 \mathrm{kN} \cdot \mathrm{m}^{-1}\right.$; (13)) or long jumping $\left(14-16 \mathrm{kN} \cdot \mathrm{m}^{-1} ;(20)\right)$. However, contrary to these studies, all participants in the one-leg jump task were found to decrease their leg stiffness by about $15 \%$ when imposed height changed from 55 to $95 \%$. Accounting for

TABLE 2. PCA results showing commonalties and factor loadings for each variable, eigenvalues, and percentage of variance explained by each rotated principal component; factor loadings lower than 0.7 were not included in the table.

\begin{tabular}{lccc} 
& \multicolumn{2}{c}{ Factor Loadings } & \\
\cline { 2 - 3 } & \multicolumn{1}{c}{$\mathbf{1}$} & $\mathbf{2}$ & Commonalities \\
\hline $\mathrm{F}_{\text {rel }}$ & -0.904 & & 0.919 \\
$\mathrm{CT}$ & 0.794 & & 0.479 \\
$\mathrm{~K}_{\text {leg }}$ & -0.750 & & 0.954 \\
$\Delta \mathrm{r}$ & & 0.883 & 0.944 \\
$\alpha$ & & & 0.276 \\
$\mathrm{D}_{\max }$ & & 0.925 & 0.641 \\
eigenvalue & 2.672 & 1.898 & \\
$\%$ of variance & 44.54 & 31.64 & \\
\hline
\end{tabular}

Abbreviations refer to maximum ground reaction force $\left(F_{\text {rel }}\right)$, contact time (CT), leg stiffness $\left(\mathrm{K}_{\text {leg }}\right)$, leg shortening $(\Delta r)$, angle swept during the contact time $(\alpha)$, and jump height $\left(D_{\text {max }}\right)$.

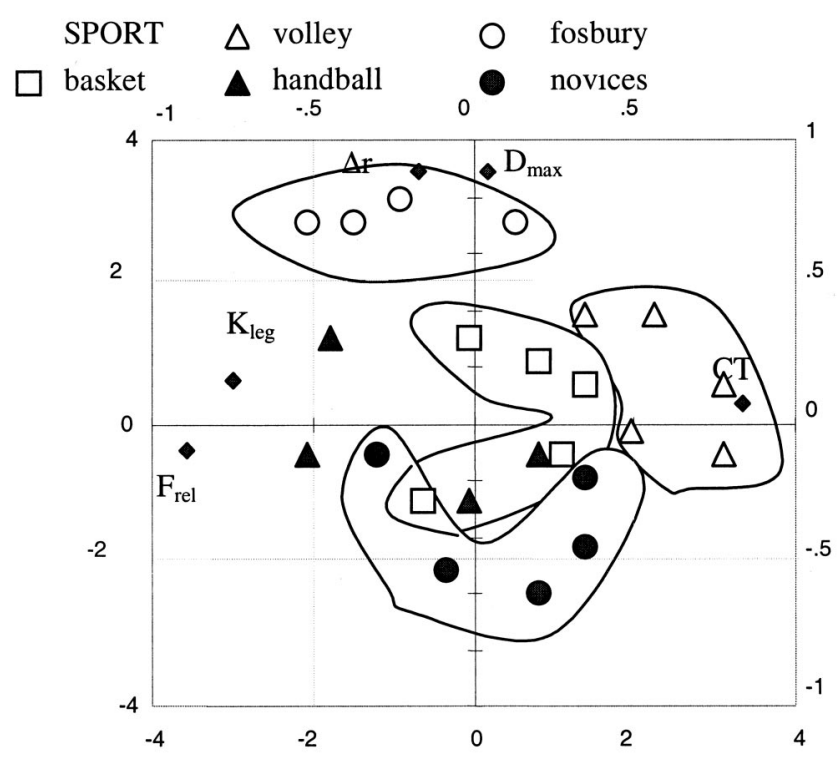

FIGURE 5-Factor scores for each participant (mean value by subject) on the two rotated principal components. The $\mathrm{x}$-axis represents the first principal component (bringing together contact time, CT; leg stiffness, $\mathrm{K}_{\mathrm{leg}}$; and vertical maximum force scaled to body mass, $\mathrm{F}_{\text {rel }}$ ), and the $y$-axis represents the second principal component (linking leg compression, $\Delta \mathrm{r}$; and jumping height, $\left.\mathrm{D}_{\max }\right)$. Left and bottom parts: numbers represent individual scores; right and upper parts: numbers represent the factor scores.

this decrease in $\mathrm{k}_{\text {leg }}$ was a small increase in maximum vertical force and a large increase in leg shortening when compared with other results. For instance, mean leg shortening was $20.4 \mathrm{~cm}$ in the present study, much greater than the value found in running $(14 \mathrm{~cm}$; (13)), in long jumping $(17 \mathrm{~cm}$; $(20))$, or in hopping in place $(8 \mathrm{~cm}$; $(12,15))$. The observed decrease in $\mathrm{k}_{\mathrm{leg}}$ with jumping height is also in contradiction with Farley et al's (9) results that indicated an increase in $\mathrm{k}_{\mathrm{leg}}$ with hopping height in the task of hopping in place. An obvious reason underlying this difference can be found in the task performed (one-leg jump vs two-leg jump). In the run-andjump task, participants must change the kinetic energy stored during the run into potential gravitational energy, and additional parameters, such as the angle swept during the ground contact phase, can be modulated for this purpose. Due to the severe constraints imposed on the running phase $(5 \mathrm{~m})$, a large amount of kinetic energy cannot be stored during that phase, yielding large contact time $(255 \mathrm{~ms})$. This in turn accentuates the eccentric phase and the shortening of the leg, preventing a large increase in vertical ground reaction force $(17,21)$. Moreover, the short running phase certainly reduces the stretch shortening cycle, and does not allow an efficient transformation of kinetic energy in potential gravitational energy (16). Thus, physiological and mechanical properties of the run-and-jump task explain the modest increase in vertical ground reaction force as well as the large increase in leg compression (1), together resulting in the observed decrease in leg stiffness with jumping height. 


\section{PCA and expertise}

Earlier studies in drop jumping and running have evidenced a negative relationship between contact time and leg stiffness $(3,13)$. This negative relationship was not directly observed in our study. The type of jump studied may explain this difference, as indicated above. However, it is also possible that other jumping parameters play a role in jumping performance and modulate the contact time-leg stiffness relationship. The PCA was used to capture this relationship among variables, and its first component indeed indicated that the negative relation between contact time and $\mathrm{k}_{\mathrm{leg}}$ was somehow crossed with a positive relation between contact time and the vertical force $\mathrm{F}_{\max }$.

Thus, the PCA appears to be an efficient method for determining which variables contribute to the behavior of leg stiffness. The PCA had another advantage on classical methods, in allowing the discrimination of jumping expertise and categories. Differences between experts and novices were observed in terms of the contribution of $k_{\text {leg }}$ to jump height, and more importantly, clear differences existed between experts in the jumping profiles.

A temporal structure of the jump was shown to be specific to volleyball players, with long impulse, low level of vertical ground reaction force, and small value of leg stiffness. The specificity of this sport could explain such a behavior: volleyball players do not produce direct confrontation, but the optical regulation of the ball's approach and of the opponent's movement is necessary to achieve an attack. This implies keeping the feet in contact with the ground for a long period of time before takeoff. The long time of impulse involves a low level of leg stiffness (3); thus, the model captures efficiently that behavior. In addition, the vertical performance of volleyball players was $65 \mathrm{~cm}$, and the value of the leg stiffness was low $\left(10.7 \mathrm{kN} \cdot \mathrm{m}^{-1}\right)$. The angle swept by the leg was constant (about $16^{\circ}$ ), independent of the target's height.

A heterogeneous and neutral profile characterized handball and basketball players, with no distinctive differences between the two components. Handball and basketball activities involve a high level of constraints, with direct confrontation between the opponents, where the jumps must be highly adaptive. However, few differences have been found between these two jumping categories. Thus, handball players used the increase of the angle swept by the leg as regulator parameter: the mean value of this angle was about $13.5^{\circ}$ in the low-target condition, and increased to $17^{\circ}$ in the high-target condition. This argues for a motor control signature specific to the sport category. On the other hand, basketball players do not show such a characteristic profile, with variables that keep very independent of the height of the target.

Fosbury athletes, in contrast, are based on the second component of the model, revealing an important leg shortening involving a good vertical performance $(75 \mathrm{~cm})$. The principal parameter used by Fosbury athletes to maximize performance was the increase in the angle swept by the leg, shifting from a mean value of $14^{\circ}$ in the low condition to $15.8^{\circ}$ in the highest condition. This behavior is very close to the one found with the handball players. This argues for a similar type of impulse task in these two sports: a running vertical one-leg jump to clear either a bar or a human defensive wall.

Lastly, novices are based on a negative value of the second component of the PCA, showing a low leg shortening associated with a low vertical performance $(58 \mathrm{~cm})$. The typical behavior of novices was a nonadaptative utilization of the impulse parameters, with a very unpredictable change of values with the increase of the target.

To conclude, this study reveals two main results. First, the leg stiffness was not used to increase the height of the jump during a short running one-leg vertical jump. Second, the analysis performed on the sport categories indicates the existence of different jumping profiles, characterized by specific, sport-related impulse parameters.

This argues for a motor control signature, as a potential trace of expertise. This result encourages us to investigate this concept of motor control signature in different types of jumps.

We would like to thank Mr. Albert Gaudin (Biometrics France) for providing the VICON motion analysis system, and Fabrice Mégrot for his help in data acquisition and processing.

The research reported in this article was supported by the French Ministère de la Recherche (Fond National pour la Science, Cognitique A118 and IUF: 2001:2006) and the University of Paris XI.

the Human Body (Report DOT-HS-801430). Wright-Patterson Air force Base, OH: Aerospace Medical Research Laboratory, Publication 22151, 1975, pp. 74-137.

7. Dapena, J. Mechanics of translation in the Fosbury-flop. Med. Sc. Sports Exerc. 12:37-44, 1980.

8. Dapena, J. Mechanics of rotation in the Fosbury-flop. Med. Sc. Sports Exerc. 12:45-53, 1980.

9. Farley, C. T., R. Blickhan, J. Saito, and C. R. Taylor. Hopping frequency in humans: a test of how springs set stride frequency in bouncing gaits. J. Appl. Physiol. 71: 2127-2132, 1991.

10. Farley, C. T., J. GlasheEn, and T. A. McMahon. Running springs: speed and animal size. J. Exp. Biol. 185:71-86, 1993.

11. Farley, C. T., H. H. P. Houdijk, C. Van Strien, and M. Louie. Mechanism of leg stiffness adjustment for hopping on surfaces of different stiffnesses. J. Appl. Physiol. 85:1044-1055, 1998. 
12. Farley, C. T., and D. C. Morgenroth. Leg stiffness depends on ankle stiffness during human hopping. J. Biomech. 32:267-273, 1999.

13. Farley, C. T., and O. GonZalez. Leg stiffness and stride frequency in human running. J. Biomech. 29:181-186, 1996.

14. Ferris, D. P., and C. T. Farley. Interaction of leg stiffness and surface stiffness during human hopping. J. Appl. Physiol. 82:1522, 1997.

15. Granata, K. P., D. A. Padua, and S. E. Wilson. Gender differences in active musculoskeletal stiffness. Part II. Quantification of leg stiffness during functional hopping tasks. J. Electromyogr. Kinesiol. 12:127-135, 2002.

16. KomI, P. V. Stretch shortening cycle: a powerful model to study normal and fatigued muscle. J. Biomech. 33:1197-1206, 2000.
17. Komi, P. V., and A. Gollhofer. Stretch reflex can have an important role in force enhancement during SSC-exercise. J. Appl. Biomech. 13:451-460, 1997.

18. McMahon, T. A., G. Valiant, and E. C. Frederick. Groucho running. J. Appl. Physiol. 62:2326-2337, 1987.

19. McMahon, T. A., and G. C. Cheng. The mechanics of running: how does stiffness couple with speed? J. Biomech. 23:65-78, 1990.

20. Seyfarth, A., A. Friedrichs, V. Wank, and R. Blickhan. Dynamics of the long jump. J. Biomech. 32:1259-1267, 1999.

21. Young, W., G. Wilson, and C. Byrne. Relationship between strength qualities and performance in standing and run-up vertical jumps. Sports Med. Phys. Fitness 39:285-293, 1999. 